\title{
Prevalência do astigmatismo e distribuição de seu eixo em pacientes de um serviço oftalmológico privado
}

\author{
Prevalence of astigmatism and its axis \\ in an ophthalmology private clinic
}

Tatiana Rocha Rayes ${ }^{1}$, Guilherme Rocha Rayes ${ }^{2}$, Felipe Eing ${ }^{3}$, Heriberto Pinto Guimarães Neto ${ }^{3}$, Francisco Azevedo Marquardt ${ }^{4}$, Assad Rayes $^{5}$

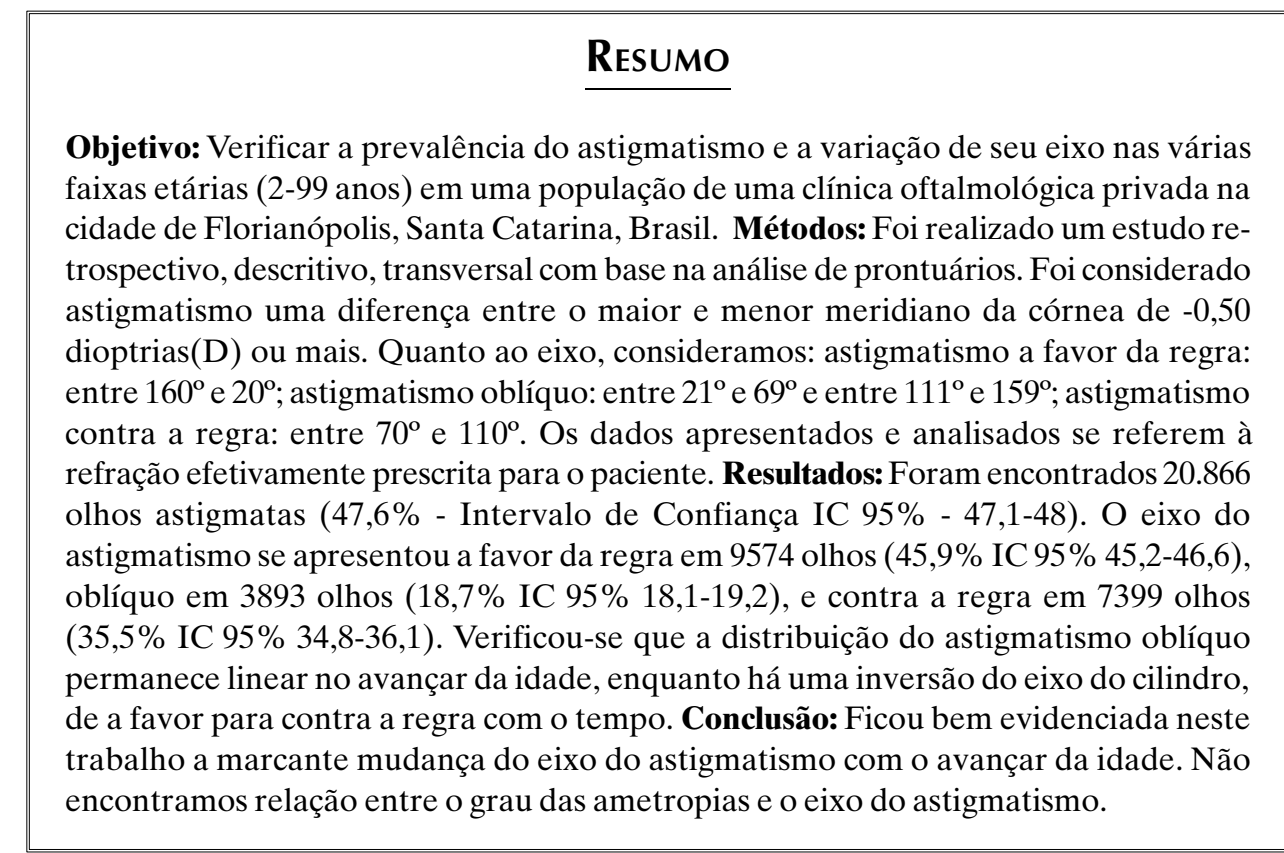

Descritores: Astigmatismo/epidemiologia; Prevalência; Distribuição por idade; Óculos; Erros de refração; Acuidade visual

\footnotetext{
${ }^{1}$ Acadêmica do $6^{\circ}$ ano de Medicina de Itajaí - UNIVALI - Itajaí (SC), Brasil;

${ }^{2}$ Acadêmico do $5^{\circ}$ ano de Medicina de Tubarão - UNISUL - Tubarão (SC), Brasil;

${ }^{3}$ Residente do $3^{\circ}$ ano em Oftalmologia do Hospital Governador Celso Ramos - Florianópolis - (SC), Brasil;

${ }^{4}$ Residente do $2^{\circ}$ ano em Oftalmologia do Hospital Governador Celso Ramos - Florianópolis - (SC), Brasil;

${ }^{5}$ Doutor em Oftalmologia pela Universidade Federal de Minas Gerais; Supervisor da Residência Médica em Oftalmologia do Hospital

Governador Celso Ramos - Florianópolis - (SC), Brasil.

Trabalho realizado no Instituto da Visão Assad Rayes, Florianópolis -(SC), Brasil.

Recebido para publicação em: 30/08/2007 - Aceito para publicação em 6/12/2007
} 


\section{INTRODUÇãO}

D enomina-se ametropia o erro da refração ocular (miopia, hipermetropia e astigmatismo), que ocasiona a perda da nitidez da imagem que teria que ser formada na retina. A não correção desses erros refrativos corresponde a maior causa de deficiência visual no mundo ${ }^{(1)}$.Apesar das ametropias poderem ser corrigidas com o uso de óculos, lentes de contato, ou cirurgia refrativa, o impacto econômico da correção dessas condições é enorme ${ }^{(2)}$.

Astigmatismo é um erro refrativo no qual a imagem não atinge a retina em um único ponto focal, mas sim, sempre, em duas linhas focais, em decorrência da presença de meridianos corneanos e ou cristalinianos de diferentes poderes dióptricos, sendo que a medida do intervalo focal entre eles corresponde ao grau do astigmatismo. Este astigmatismo é conhecido como astigmatismo regular e é corrigido por lentes cilíndricas. Em casos de doenças corneanas ou cristalinianas, podem vir a ocorrer irregularidades grosseiras na curvatura destes meridianos, que não são compensadas com lentes cilíndricas. Este astigmatismo é conhecido como astigmatismo irregular.

No astigmatismo regular, os meridianos de maior e menor poder da córnea estão sempre colocados perpendicularmente, ou seja, a $90^{\circ}$. Se um meridiano de menor curvatura estiver em um ângulo entre $160^{\circ} \mathrm{e}$ $20^{\circ}$ com o plano horizontal, chamamos de astigmatismo a favor da regra; entre $70^{\circ}$ e $110^{\circ}$, astigmatismo contra a regra; e entre $21^{\circ}$ e $69^{\circ}$ e entre $111^{\circ}$ e $159^{\circ}$, astigmatismo oblíquo.

Historicamente, tem sido descrito que o eixo do astigmatismo sofre variações com a idade. Em crianças e adultos jovens, o astigmatismo a favor da regra é preponderante, e com o avançar da idade essa preponderância torna-se cada vez menor até que, por volta dos 50 anos, o astigmatismo oblíquo torna-se 3 vezes mais comum e o contra a regra, 5 a 6 vezes; em torno dos 80 anos, o astigmatismo contra a regra é uma constante ${ }^{(3)}$.

Este trabalho tem como objetivo verificar a prevalência do astigmatismo e a variação de seu eixo nas várias faixas etárias (2-99 anos) em uma população de uma clínica oftalmológica privada na cidade de Florianópolis - SC.

\section{Métodos}

Foi realizado um estudo transversal, retrospectivo, descritivo, com base na análise de prontuários de pa- cientes entre 2 e 99 anos de idade, atendidos no Instituto da Visão Assad Rayes, em Florianópolis - SC, no período de 10 de junho de 1996 a 3 de fevereiro de 2007.

Todos os pacientes foram examinados pelo mesmo médico oftalmologista. Para a refratometria, utilizou-se refração dinâmica em pacientes com 40 anos ou mais, e refração estática - sob cicloplegia com tropicamida a $1 \%, 3$ vezes de 5 em $5 \mathrm{~min}$, com exame após $30 \mathrm{~min}$ - naqueles com menos de 40 anos.

As medidas da acuidade visual foram tomadas através de projeção de optotipos (tabela de Snellen) a 6 metros de distância (projetor de optotipos American Optical $\left.{ }^{\circledR}\right)$. Estas medidas foram tomadas, com e sem correção óptica, individualmente, em cada olho. A correção óptica foi obtida com uso do refrator (Bobes ${ }^{\circledR}$, Espanha, modelo AO).

Foi considerado astigmatismo uma diferença entre o maior e menor meridiano da córnea de -0,50 dioptrias(D) ou menos. Dividimos o astigmatismo quanto ao grau: astigmatismo baixo: $-0,50$ a $-1,0(\mathrm{D})$; astigmatismo médio: $-1,25$ a -3,0(D); astigmatismo alto: d"-3,25(D). Quanto ao eixo, consideramos: astigmatismo a favor da regra (AFR): eixo entre $160^{\circ}$ e $20^{\circ}$; astigmatismo oblíquo(AO): eixo entre $21^{\circ}$ e $69^{\circ}$ e entre $111^{\circ}$ e $159^{\circ}$; astigmatismo contra a regra(ACR): eixo entre $70^{\circ}$ e $110^{\circ}$.

Os dados apresentados e analisados se referem à refração efetivamente prescrita para o paciente. A análise estatística foi feita através de métodos descritivos e a avaliação da significância das correlações estatísticas foi feita através do teste do qui-quadrado, sendo considerado $\mathrm{p}<0.05$ como significativo.

\section{Resultados}

Foram examinados prontuários de 21.940 pacientes, perfazendo um total de $43.880 \mathrm{olhos}$. A maioria da amostra $(29.030$ olhos - 66,2\% - IC 95\% 65,70-66,60) era de pacientes do sexo feminino e 14850 olhos (33,8\% - IC $95 \%$ 33,3-34,2) de pacientes masculinos. A distribuição dos pacientes de acordo com a idade está ilustrada no gráfico $1 . \mathrm{O}$ paciente mais jovem possuía 2 anos e o mais idoso 99 anos, sendo que a média de idade da amostra foi de 40,4 anos.

Foram encontrados 20.866 olhos astigmatas (47,6\% - IC 95\% 47,1-48). A prevalência do astigmatismo dentre as faixas etárias pode ser observada no gráfico 2. A maioria dos olhos com astigmatismo $(13.407-64,3 \%$ IC95\% 63,6-64,9) era de pacientes do sexo feminino e 7.459 olhos (35,7\% - IC95\% 35,1-36,4), do sexo masculino. 


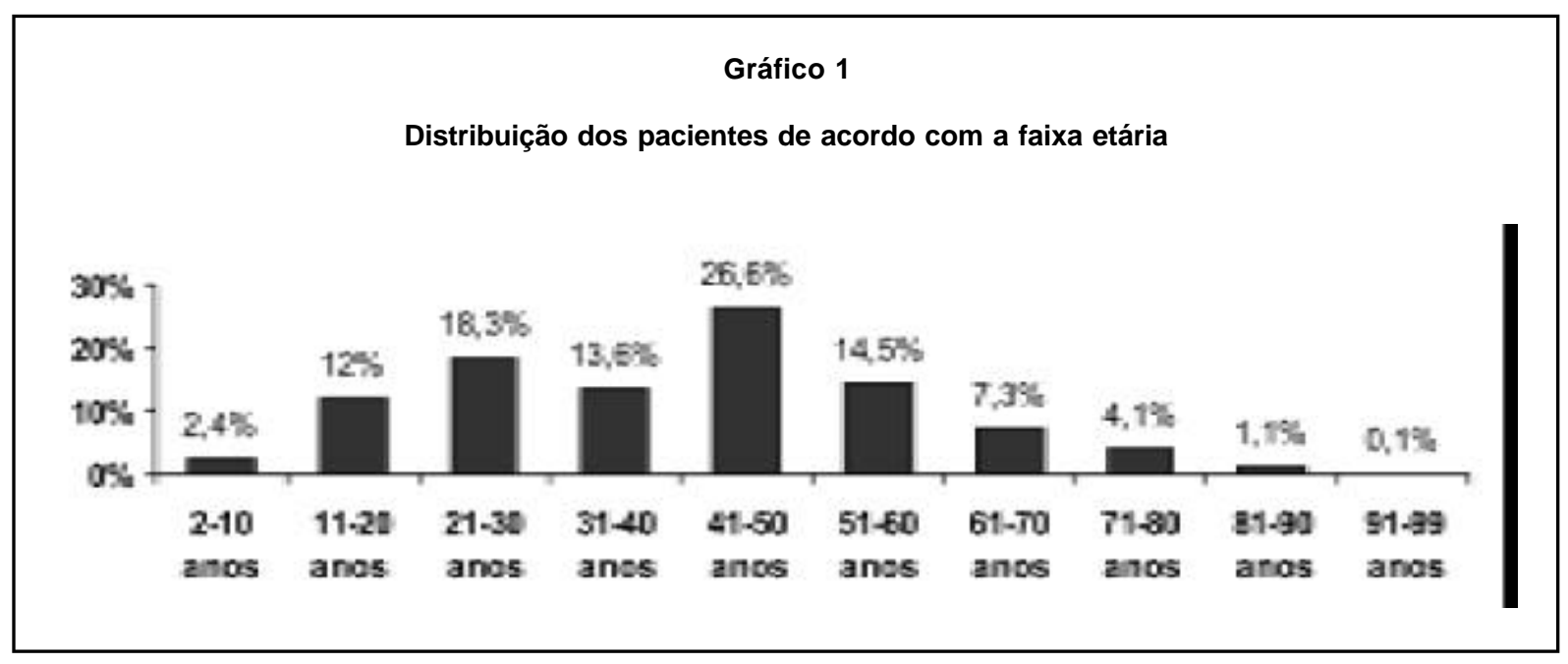

\section{Gráfico 2}

Prevalência de astigmatismo nas diversas faixas etárias

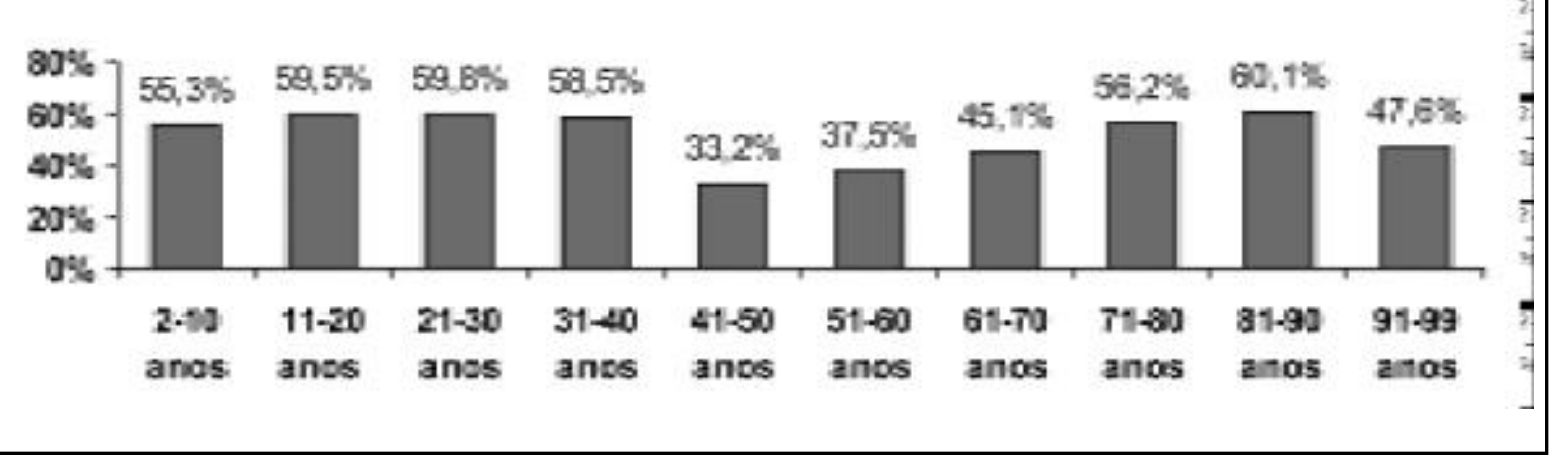

Com relação ao grau, estes olhos foram distribuídos em astigmatismo baixo em $14.154(67,8 \%$ IC 95\% 67,2-68,5), médio em 6010 (28,8\% - IC 95\% 28,2-29,4), e alto em 700 (3,4\% - IC 95\% 3,1-3,6), (Gráfico 3).

Houve significância estatística $(\mathrm{p}>0,00001)$ na distribuição de freqüência entre o grau do astigmatismo nas diversas faixas etárias (Gráfico 4).

Com relação ao eixo do astigmatismo, em 9.574 olhos (45,9\% IC 95\% 45,2-46,6) este se apresentou a favor da regra, em 3.893 olhos (18,7\% IC 95\% 18,119,2) oblíquo, e em 7.399 olhos (35,5\% IC 95\% 34,8$36,1)$ contra a regra - Gráfico 5.

Não foi estatisticamente significativa $(p>0,05)$ a distribuição da freqüência entre os sexos masculi-

\section{Gráfico 3}

Distribuição dos olhos astigmatas de acordo com a presença e o grau do astigmatismo

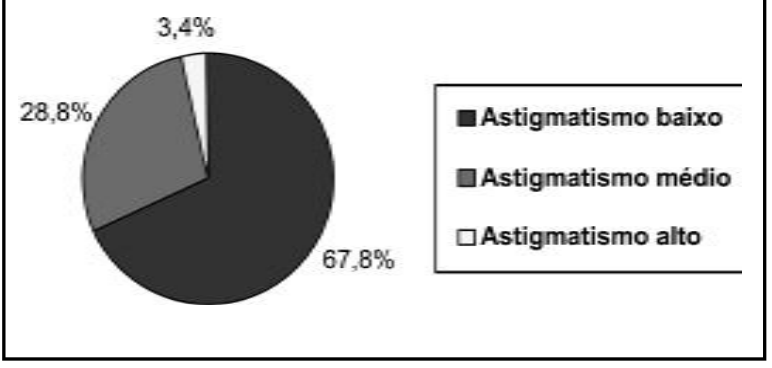




\section{Gráfico 4}

Distribuição dos olhos de acordo com o grau do astigmatismo por faixa etária $(p<0.0001)$

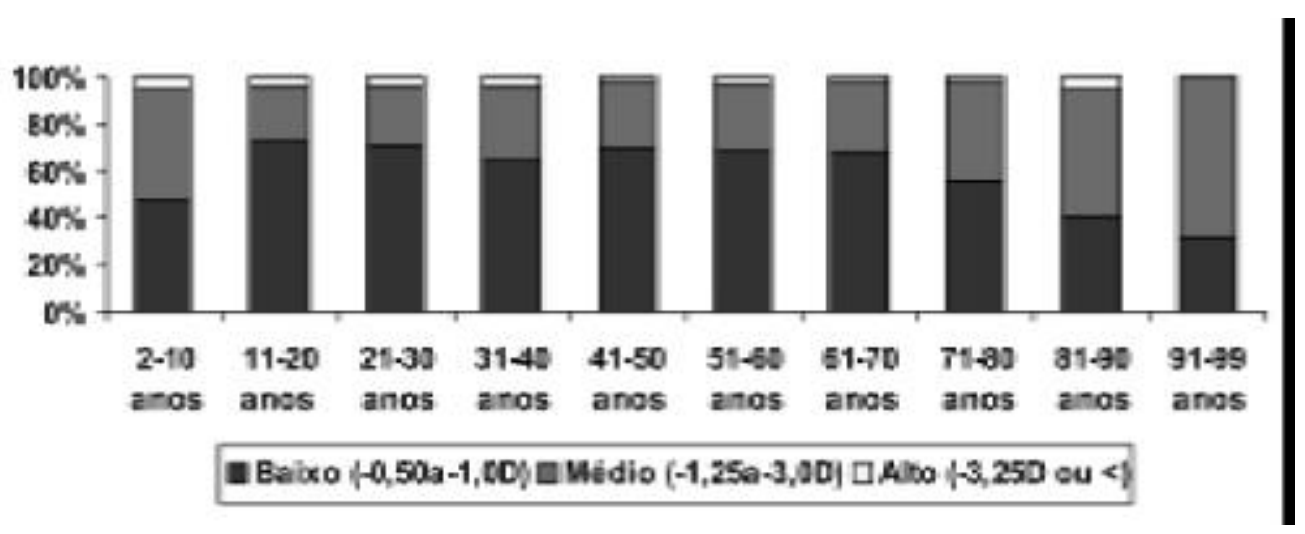

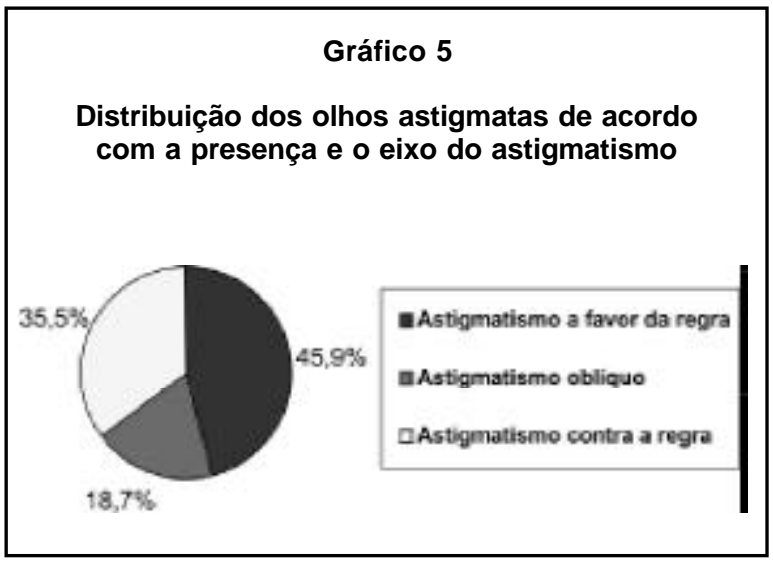

no e feminino e a presença do astigmatismo, seu grau e seu eixo.

Houve correlação estatisticamente significativa na distribuição do eixo do astigmatismo entre as diversas faixas etárias $(\mathrm{p}<0,00001)$ - Tabela 1.

Observou-se que a distribuição do astigmatismo oblíquo permanece linear no avançar da idade, enquanto há uma inversão do eixo do cilindro, de a favor para contra a regra com o tempo (Gráfico 6).

Não houve diferença estatisticamente significativa na distribuição do eixo do astigmatismo entre os sexos masculino e feminino $(\mathrm{p}>0,05)$.

Também não se observou relação estatisticamente significante entre a distribuição do grau do astigmatismo, miopia e hipermetropia e o eixo encontrado no astigmatismo $(p>0,05)$.

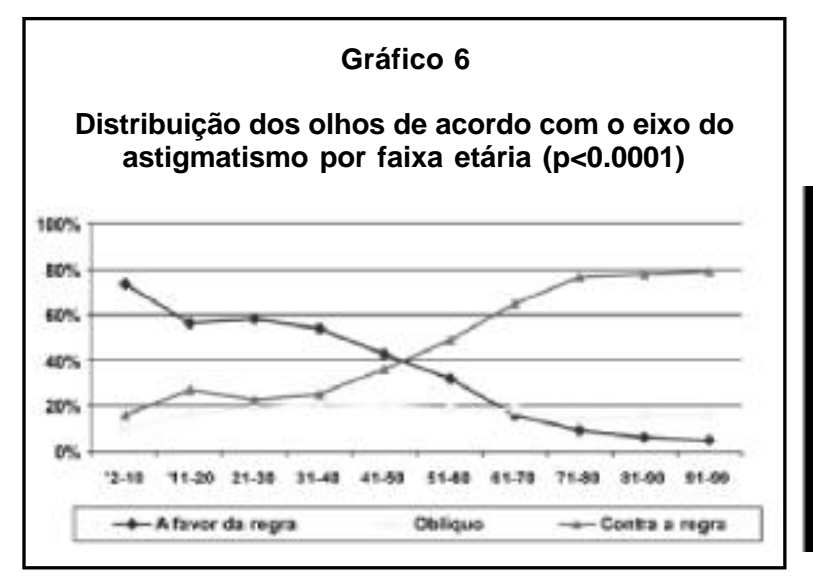

\section{Discussão}

O astigmatismo é um erro refrativo bastante comum na prática médica. Apresentamos em nossa amostra a prevalência desta ametropia, a variação do grau e do eixo do astigmatismo e suas correlações com o sexo e faixa etária.

A prevalência geral de astigmatismo na população estudada foi $47,6 \%$, concordando com a taxa de $50.2 \%$ relatada em um estudo realizado com 4565 iranianos ${ }^{(4)}$.

Nossa prevalência se mostra um pouco superior ao encontrado no Blue Mountains Eye Study ${ }^{(5)}$, que estudou os erros refrativos de 3.654 australianos entre 4997 anos e encontrou uma prevalência de $37 \%$, e ao descrito no National Blindness and Low Vision Survey of 
Tabela 1

Distribuição dos olhos de acordo com o eixo do astigmatismo por faixa etária $(p<0.0001)$

\begin{tabular}{|c|c|c|c|c|c|}
\hline \multicolumn{2}{|c|}{$\begin{array}{c}\text { Eixo do astigmatismo } \\
x \\
\text { Faixa Etária }\end{array}$} & \multirow{2}{*}{$\begin{array}{c}\begin{array}{c}\text { A favor } \\
\text { da regra }\end{array} \\
469\end{array}$} & \multirow{2}{*}{\begin{tabular}{|c|} 
Oblíquo \\
67
\end{tabular}} & \multirow{2}{*}{$\begin{array}{c}\text { Contra } \\
\text { a regra }\end{array}$} & \multirow{2}{*}{$\begin{array}{c}\text { Total } \\
638\end{array}$} \\
\hline 2-10 anos & $\mathrm{n}$ & & & & \\
\hline & $\%$ & 73,5 & 10,5 & 16,0 & 100,0 \\
\hline \multirow[t]{2}{*}{$11-20$ anos } & $\mathrm{n}$ & 1654 & 481 & 779 & 2914 \\
\hline & $\%$ & 56,7 & 16,5 & 26,8 & 100,0 \\
\hline \multirow[t]{2}{*}{$21-30$ anos } & $\mathrm{n}$ & 2799 & 915 & 1095 & 4809 \\
\hline & $\%$ & 58,20 & 19,0 & 22,8 & 100,0 \\
\hline \multirow[t]{2}{*}{$31-40$ anos } & $\mathrm{n}$ & 1885 & 719 & 887 & 3491 \\
\hline & $\%$ & 54,0 & 20,6 & 25,4 & 100,0 \\
\hline \multirow[t]{2}{*}{$41-50$ anos } & $\mathrm{n}$ & 1664 & 794 & 1410 & 3868 \\
\hline & $\%$ & 43,0 & 20,5 & 36,5 & 100,0 \\
\hline \multirow[t]{2}{*}{$51-60$ anos } & $\mathrm{n}$ & 756 & 457 & 1168 & 2381 \\
\hline & $\%$ & 31,8 & 19,2 & 49,0 & 100,0 \\
\hline \multirow[t]{2}{*}{$61-70$ anos } & $\mathrm{n}$ & 233 & 269 & 945 & 1447 \\
\hline & $\%$ & 16,1 & 18,6 & 65,3 & 100,0 \\
\hline \multirow[t]{2}{*}{$71-80$ anos } & $\mathrm{n}$ & 96 & 143 & 778 & 1017 \\
\hline & $\%$ & 9,4 & 14,0 & 76,6 & 100,0 \\
\hline \multirow[t]{2}{*}{$81-90$ anos } & $\mathrm{n}$ & 17 & 45 & 220 & 282 \\
\hline & $\%$ & 6,0 & 16,0 & 78,0 & 100,0 \\
\hline \multirow[t]{2}{*}{ 91-99 anos } & $\mathrm{n}$ & 1 & 3 & 15 & 19 \\
\hline & $\%$ & 5,2 & 15,8 & 79,0 & 100,0 \\
\hline Total dos & $\mathrm{n}$ & 9574 & 3893 & 7399 & 20866 \\
\hline astigmatismos & $\%$ & 45,9 & 18,6 & 35,5 & 100,0 \\
\hline
\end{tabular}

Bangladesh ${ }^{(6)}$ que relatou uma taxa de $34,6 \%$ de astigmatismo entre 3.625 pessoas com 30 anos ou mais.

Entretanto, outros estudos encontraram valores ainda menores relatando uma prevalência de astigmatismo de $20 \%$ entre pacientes adultos entre $16-85$ anos na Dinamarca ${ }^{(7)}$ e outro estudo finlandês, que encontrou uma taxa de $10 \%$ com pessoas entre $6-85$ anos ${ }^{(8)}$.

Acreditamos que a maior prevalência de astigmatismo em nossa amostra tenha ocorrido por nossa população ser proveniente de uma clínica oftalmológica, onde supostamente encontraremos um maior número de pessoas com deficiências visuais.

Diferentemente do relatado em outros estudos ${ }^{(2,59-10)}$, a prevalência de astigmatismo não aumentou com o avançar da idade, permanecendo quase que constante nas diversas faixas etárias. Muito provavelmente, este fato deve estar relacionado com o perfil das populações estudadas; enquanto, naqueles trabalhos foram feitos estudos randomizados junto à população geral, nosso estudo foi realizado com amostra de conveniência, composta de pacientes que procuravam a clínica com queixas visuais.

Apesar de termos encontrado uma maior parcela de mulheres com astigmatismo (64,3\%), este achado não é significativo, uma vez que a maioria de nossa amostra $(66,2 \%)$ era composta de pacientes do sexo feminino. Desta forma, nosso resultado concorda com vários outros trabalhos que também não encontraram diferença na prevalência do astigmatismo entre os sexos ${ }^{(5-6,11-13)}$.

Com relação ao eixo do astigmatismo, encontramos na população geral $45,9 \%$ de astigmatismo a favor da regra (AFR), 35,5\% contra a regra (ACR) e 18,7\% oblíquo (AO), diferindo um pouco do relatado por um estudo realizado em uma população iraniana: $33,6 \%$ a AFR, 36,9\% ACR e 29,3\% $\mathrm{AO}^{(4)}$.

A distribuição do eixo nos pacientes acima de 30 anos (AFR: 20,2\%, ACR: 51,6\%, AO: 29,2\%) é similar 
ao encontrado em pacientes da mesma faixa etária no National Blindness and Low Vision Survey of Bangladesh, que relatou uma prevalência de AFR de 12,1\%, ACR: $58,7 \%$, AO: $29,3 \%)^{(6)}$.

Nas crianças entre 2 e 10 anos, o eixo do astigmatismo teve a seguinte distribuição: AFR: 73,5\%, ACR:16\%, AO: $10,5 \%$; estando entre o que foi encontrado em um estudo com crianças australianas de 12 anos (AFR: 40,4\%, ACR: 43,6\%, AO: $16 \%)^{(14)}$ e um estudo chinês com crianças de $6 \mathrm{e}$ 7 anos (AFR: 90,34\%, ACR:0,14\%, AO: 9,25\%) ${ }^{15}$.

Assim como, em diversos outros estudos, encontramos uma variação do eixo do astigmatismo com a idade, sendo que há uma tendência do astigmatismo se tornar contra a regra com o avançar da idade ${ }^{(5,7,16-19)}$.

Não encontramos relação entre o eixo do astigmatismo e o grau das ametropias esféricas como foi relatado em um trabalho do Reino Unido ${ }^{(19)}$.

As causas pelas quais ocorre uma alteração do eixo do astigmatismo de AFR para ACR ainda não estão totalmente aceitas ou esclarecidas, sendo que a mais provável seria a pressão que as pálpebras superiores exercem sobre a córnea, como por exemplo nas dermatocálases, tumores palpebrais, calázios, etc. Especula-se também o papel da contração do músculo ciliar, que além de com a idade se tornar incapaz de alterar a forma do cristalino, influenciaria na curvatura corneana.

Outra hipótese seria a de que as porções superior e inferior da córnea receberiam menos aporte nutricional, devido ao fato de que se encontram localizadas mais distante das artérias ciliares posteriores longas, e em conseqüência disto ficariam mais enfraquecidas estruturalmente, alterando a curvatura da mesma.

De qualquer modo, ficou bem evidenciada neste trabalho a marcante mudança do eixo do astigmatismo de a favor da regra para contra a regra com o avançar da idade.

\section{Abstract}

Purpose: to determine the prevalence of astigmatism and the change of the axis according to the age in an ophthalmology private clinic population in the city of Florianópolis, Santa Catarina, Brazil. Methods: A retrospective, descriptive, cross-sectional study was carried out based on the analyses of medical files. Astigmatism was classified as a cylindrical equivalent $<-0,5$ diopter (D). Astigmatism with the rule was defined as $160^{\circ}$ to $20^{\circ}$, oblique astigmatism as $21^{\circ}$ to $69^{\circ}$ and $111^{\circ}$ to $159^{\circ}$, and astigmatism against the rule when axes were located from $70^{\circ}$ to $110^{\circ}$. The basis were presented and analyzed according to the glasses prescription. Results: There were found 20866 astigmatic eyes (47,6\% - 95\% Confidence Interval CI - 47,1-48). The astigmatism axis was with the rule in 9574 eyes (45,9\% CI 95\% 45,2-46,6), oblique in 3893 eyes $(18,7 \%$ CI 95\% 18,1-19,2), and against the rule in 7399 eyes $(35,5 \%$ CI 95\% 34,8-36,1). It was verified the prevalence of the oblique axis remains stable with the age, while there is an inversion in the cylindric axis from with the rule to against the rule as time goes by. Conclusion: This study has made evident the change in the axis according to the age. It was not found any relation between the degrees of the ametropy and the axis of the astigmatism.

Keywords: Astigmatism/epidemiology; Prevalence; Age distribution; Eyeglasses; Refractive errors; Visual acuity

\section{RefERÊNCIAS}

1. Dandona R, Dandona L. Refractive error blindness. Bull World Health Organ. 2001; 79(3):237-43. Review.

2. Katz J, Tielsch JM, Sommer A. Prevalence and risk factors for refractive errors in an adult inner city population. Invest Ophthalmol Vis Sci. 1997; 38(2):334-40.

3. Jackson E. J Amer Med Assoc. 1932; 98:132 apud Duke-Elder S. System of ophthalmology. St Louis: Mosby; 1970. v.5, p. 282.

4. Hashemi H, Hatef E, Fotouhi A, Mohammad K. Astigmatism and its determinants in the Tehran population: the Tehran eye study. Ophthalmic Epidemiol. 2005; 12(6):373-81.

5. Attebo K, Ivers RQ, Mitchell P. Refractive errors in an older population: the Blue Mountains Eye Study. Ophthalmology. 1999; 106(6):1066-72.

6. Bourne RR, Dineen BP, Ali SM, Noorul Huq DM, Johnson GJ. Prevalence of refractive error in Bangladeshi adults: results of the National Blindness and Low Vision Survey of Bangladesh. Ophthalmology. 2004; 111(6):1150-60.

7. Fledelius HC. Prevalences of astigmatism and anisometropia in adult danes. With reference to presbyopes' possible use of supermarket standard glasses. Acta Ophthalmol (Copenh). 1984; 62(3):391-400.

8. Aine E. Refractive errors in a Finnish rural population. Acta Ophthalmol (Copenh). 1984; 62(6):944-54.

9. Wong TY, Foster PJ, Hee J, Ng TP, Tielsch JM, Chew SJ, et al. Prevalence and risk factors for refractive errors in adult Chinese in Singapore. Invest Ophthalmol Vis Sci. 2000; 41(9):2486-94.

10. Gudmundsdottir E, Jonasson F, Jonsson V, Stefánsson E, Sasaki $\mathrm{H}$, Sasaki K. "With the rule" astigmatism is not the rule in the elderly. Reykjavik Eye Study: a population based study of refraction and visual acuity in citizens of Reykjavik 50 years and older. Iceland-Japan Co-Working Study Groups. Acta Ophthalmol Scand. 2000; 78(6):642-6. 
11. Wu HM, Seet B, Yap EP, Saw SM, Lim TH, Chia KS. Does education explain ethnic differences in myopia prevalence? A population-based study of young adult males in Singapore. Optom Vis Sci. 2001; 78(4):234-9.

12. Mehra V, Minassian DC. A rapid method of grading cataract in epidemiological studies and eye surveys. Br J Ophthalmol. 1988; 72(11):801-3.

13. McKendrick AM, Brennan NA. Distribution of astigmatism in the adult population. J Opt Soc Am A Opt Image Sci Vis. 1996; 13(2):206-14.

14. Huynh SC, Kifley A, Rose KA, Morgan IG, Mitchell P. Astigmatism in 12-year-old Australian children: comparisons with a 6-year-old population. Invest Ophthalmol Vis Sci. 2007; 48(1):73-82.

15. Gong C, Chen X, Gong H. [Astigmatism analysis on 983 astigmatic eyes between six to seven years children] Yan Ke Xue Bao. 2004; 20(2):104-6. Chinese.

16. Saunders H. A longitudinal study of the age-dependence of human ocular refraction - I. Age-dependent changes in the equivalent sphere. Ophthalmic Physiol Opt. 1986; 6(1):39-46.
17. Hirsch MJ. Changes in astigmatism after the age of forty. Am J Optom Arch Am Acad Optom. 1959; 36: 395-405.

18. Saunders H. Age-dependence of human refractive errors. Ophthalmic Physiol Opt. 1981; 1(3):159-74.

19. Farbrother JE, Welsby JW, Guggenheim JA. Astigmatic axis is related to the level of spherical ametropia. Optom Vis Sci. 2004; 81(1):18-26.

ENDEREÇO PARA CORRESPONDÊNCIA:

Assad Rayes

Rua Dom Joaquim, 885, $8^{\circ}$ andar

Ed. Celso Ramos Medical Center - Centro

CEP 88015-310 - Florianópolis - SC

Email: institutoassadrayes@uol.com.br 